\title{
Change of respiratory mechanics at different intra-abdominal pressures and position change during laparoscopic surgery
}

\author{
Kyu-Yong Jang, Seok Joo Hong, Hyun Kyo Lim, and Dea Ja Um \\ Department of Anesthesiology and Pain Medicine, Yonsei University Wonju College of Medicine, Wonju, Korea
}

Laparoscopy results in less scar formation due to smaller incision, and less postoperative pain, facilitating earlier ambulation. These characteristics result in decreased pulmonary complication rate and shortened admission time, leading to numerous advantages such as reduction of medical cost. As laparoscopy involves maximal increase in abdominal pressure through continuous flow of carbon dioxide gas into the abdominal cavity, numerous studies have reported the resulting hemodynamic, respiratory and endocrinologic alterations, as well as surgical complications [1]. We have compared the dynamic compliance, peak airway pressure and respiratory resistance, as the abdominal pressure and body position change during laparoscopic gynecologic surgeries.

This protocol involved 50 patients who were classified as having physical status class I or II according to the American Society of Anesthesiologists, and had no underlying cardiopulmonary diseases or planned elective operations. All patients informed consent prior to being included in the study. All patients were premedicated by glycopyrrolate $(0.004 \mathrm{mg} / \mathrm{kg})$ and midazolam $(0.06 \mathrm{mg} / \mathrm{kg}) 30$ minutes prior to induction. Anesthesia was induced by intravenous thiopental sodium (5 $\mathrm{mg} / \mathrm{kg}$ ) and vecuronium $(0.1 \mathrm{mg} / \mathrm{kg})$. Anesthesia was maintained by volume-controlled ventilation with $\mathrm{O}_{2}(2 \mathrm{~L} /$ $\min ), \mathrm{N}_{2} \mathrm{O}(2 \mathrm{~L} / \mathrm{min})$ and enflurane (1.5-2 vol\%). Additional vecuronium $(0.02 \mathrm{mg} / \mathrm{kg})$ was administered to maintain constant muscle paralysis as needed. Tidal volume of $10 \mathrm{ml} /$ $\mathrm{kg}$ and respiratory rate of $14 /$ minute was maintained with a volume anesthesia ventilator (Ohmeda, Modulus ${ }^{\circledR}$ ). Blood pressure, heart rate, oxygen saturation, and end-tidal carbon dioxide pressure $\left(\mathrm{EtCO}_{2}\right)$ were measured continuously using patient-monitoring devices. The patients were maintained in $15^{\circ}$ lithotomy-Trendelenburg position during operation time, and carbon dioxide was injected at the start of and during operation to achieve and maintain steady abdominal pressure at $14 \mathrm{mmHg}$ for $\mathrm{H}$ group and $10 \mathrm{mmHg}$ for L group. Dynamic compliance, peak airway pressure, and respiratory resistance were measured with VENTRAK ${ }^{\mathrm{TM}}$ (Novametrix Medical System Inc, USA) at 2 minutes after the beginning of mechanical ventilation (control), 3 minutes after position change (immediately before carbon dioxide insufflation) and 5 and 15 minutes after carbon dioxide insufflation. All measurements are presented as mean \pm standard deviation, and Wilcoxon signed-rank test and Mann-Whitney test were performed for intra- and inter-group comparison of respiratory dynamics. $\mathrm{P}$ values less than 0.05 were determined as statistically significant.

Dynamic compliance in the $\mathrm{H}$ group significantly decreased from $61.3 \pm 13.2 \mathrm{ml} / \mathrm{cmH}_{2} \mathrm{O}$ for the control to $57.7 \pm 11.6 \mathrm{ml} /$ $\mathrm{cmH}_{2} \mathrm{O}$ after positional change $(\mathrm{P}<0.05)$, continuing to decrease to $32.5 \pm 9.2 \mathrm{ml} / \mathrm{cmH}_{2} \mathrm{O}$ and $30.3 \pm 6.6 \mathrm{ml} / \mathrm{cmH}_{2} \mathrm{O}$ at 5 minutes and 15 minutes after carbon dioxide insufflation. Dynamic compliance also significantly decreased in the $\mathrm{L}$ group at each measurement. There was no significant difference between the $\mathrm{H}$ group and $\mathrm{L}$ group in dynamic compliance at any measurement point (Fig. 1). In the H group, the peak airway pressure did not change noticeably upon positional change compared to the control, but was significantly elevated with an increasing trend with the passage of time. In the L group, the peak airway pressure was $13.1 \pm 2.1 \mathrm{cmH}_{2} \mathrm{O}$ for the control, which

Corresponding author: Dea Ja Um, M.D., Department of Anesthesiology and Pain Medicine, Yonsei University Wonju College of Medicine, 162, Ilsan-dong, Wonju 220-701, Korea. Tel: 82-33-741-1522, Fax: 82-33-742-8198, E-mail: umdj@yonsei.ac.kr

(c) This is an open-access article distributed under the terms of the Creative Commons Attribution Non-Commercial License (http:// creativecommons.org/licenses/by-nc/3.0/), which permits unrestricted non-commercial use, distribution, and reproduction in any medium, provided the original work is properly cited. 


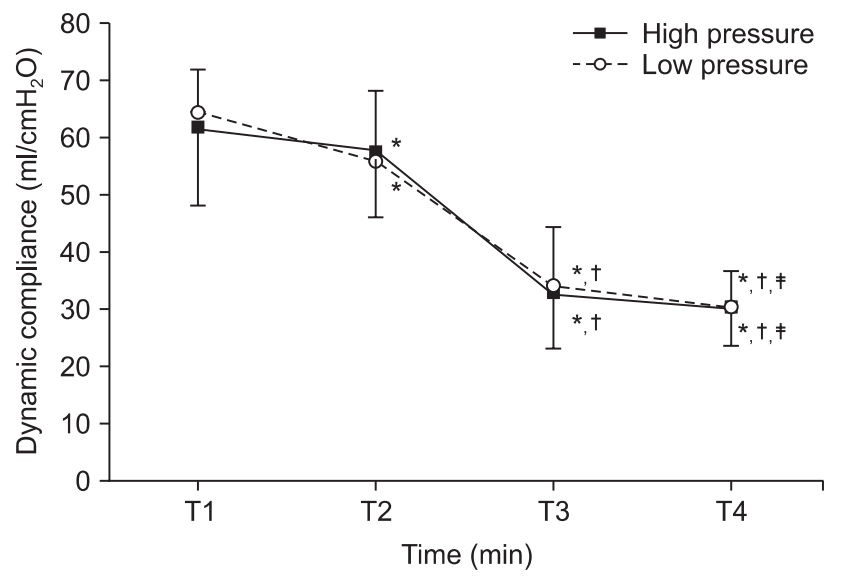

Fig. 1. Dynamic compliance during laparoscopic surgery. Values are expressed as mean $\pm \mathrm{SD}$. ${ }^{*} \mathrm{P}<0.05$ when compared with $\mathrm{T} 1 .{ }^{\dagger} \mathrm{P}<$ 0.05 when compared with $\mathrm{T} 2 .{ }^{\ddagger} \mathrm{P}<0.05$ when compared with T3. T1: After $2 \mathrm{~min}$ from the beginning of mechanical ventilation, T2: $3 \mathrm{~min}$ after position change, T3: 5 min after $\mathrm{CO}_{2}$ insufflation, T4: $15 \mathrm{~min}$ after $\mathrm{CO}_{2}$ insufflation.

significantly increased to $14.2 \pm 3.2 \mathrm{cmH}_{2} \mathrm{O}$ after positional change. The respiratory resistance was not significantly different between the two groups at any measurement point.

The functional residual volume and lung compliance is reduced by $20 \%$ upon general anesthesis, with the reduction reaching up to $50 \%$ in the case of obesity. When positioned in the Trendelenburg position, the contents of the abdominal cavity shifted toward the head, resulting in poorer functional residual volume, total lung volume, and lung elasticity. Altered functional residual volume led to changes in the character of the lung and the chest wall, which then changed the lung compliance and resistance. Lee et al. [2] reported no difference in the peak airway pressure measured in Trendelenburg and reverse Trendenlenburg positions, while transrespiratory resistance higher in $15^{\circ}$ Trendelenburg position compared to $10^{\circ}$ reverse Trendelenburg position. However, neither group in our study demonstrated a significant increase in airway resistance after positional change. Kim et al. [3] reported that compared to pelviscopy performed at $10^{\circ}$ Trendelenburg position, laparoscopic surgery performed at $10^{\circ}$ reverse Trendelenburg position resulted in smaller change in peak airway pressure and lung compliance, suggesting that this may be due to the nature of the reverse Trendelenburg position offsetting the increase in abdominal pressure. In our study, the lung compliance of two groups in the Trendelenburg position decreased by $5.9 \%$ and $13.8 \%$, and further decreased by $50.6 \%$ and $52.9 \%$ when abdominal pressure was maintained at $14 \mathrm{mmHg}$ and 10 $\mathrm{mmHg}$, respectively. Increased abdominal pressured caused by the Trendelenburg position and pneumoperitoneum decreases the functional residual volume even further, and when under mechanical ventilation receiving same tidal volume, leads to change in airway resistance due to increased airway pressure and transpulmonary pressure. Schleifer et al. [4] argued that peak airway pressure rises along with increase in abdominal pressure in laparoscopic gynecologic surgery. Bannister et al. [5] stated that increased abdominal pressure is associated with increased peak airway pressure and decreased lung compliance. This study first attempted to randomize the patients into two groups and apply different degrees of abdominal pressure, 10 $\mathrm{mmHg}$ and $14 \mathrm{mmHg}$. Different abdominal pressures were applied after dividing the patients based on their body weight, from which a comparison of the effect of abdominal pressure was made. Similar results may be expected for fully randomized patient groups with different abdominal pressures applied. Considering that the respiratory mechanism is not different between the two groups, an adequate abdominal pressure according to body weight is believed to be more appropriate than applying a uniform abdominal pressure regardless of body weight, as long as sufficient operating view is obtained.

In conclusion, different abdominal pressures applied to two patient groups undergoing gynecologic laparoscopic surgery did not result in a significant difference in measurements of dynamic compliance, peak airway pressure, and airway resistance in this study.

\section{References}

1. Joshi GP. Complications related to abdominal surgery with an emphasis on laparoscopy. Anesthesia and perioperative complications. 2nd ed. Edited by Benumof JL, Saidman LJ: Philadelphia, Mosby. 1999, pp 665-85.

2. Lee KS, Kim SY, Kim HD. Effects of intraabdominal CO2 gas insufflation on PaCO2 and tidal volume during laparoscopic surgery under general anesthesia. Korean J Anesthesiol 1992; 25: 935-40.

3. Kim JE, Nam YT, Chae YH. The effect of the body position and CO2 gas insufflation on airway pressure and compliance in normal subjects during laparoscopy or pelviscopy. Korean J Anesthesiol 1999; 36: 802-7.

4. Schleifer W, Bissinger U, Guggenberger H, Heuser D. Variance of cardiorespiratory parameters during gynaecological surgery with CO2-pneumoperitoneum. Endosc Surg Allied Technol 1995; 3: 16770.

5. Bannister CF, Brosius KK, Wulkan M. The effect of insufflation pressure on pulmonary mechanics in infants during laparoscopic surgical procedures. Paediatr Anaesth 2003; 13: 785-9. 